\title{
Nuevos Registros de XYlariales (Ascomycota) para la ArgentinA
}

\author{
ESTEBAN B. SIR ${ }^{1}$, PATRICIA V. SILVA², CAROLINA A. ROBLES ${ }^{3}$ y M. VICTORIA VIGNALE ${ }^{3}$
}

\begin{abstract}
Summary: New records of Xylariales (Ascomycota) in Argentina. Camillea scriblita, and Nemania immersidiscus are reported for the first time for Argentinean mycobiota. Descriptions and photographs of both species, and of the recently reported Phylacia turbinata are included. In addition, a map showing the distribution based on their records in the Neotropics is presented.
\end{abstract}

Key words: Camillea, Nemania, Mycogeography.

\begin{abstract}
Resumen: Camillea scriblita y Nemania immersidiscus son registradas por primera vez para la micobiota Argentina. Se incluyen descripciones y fotografias de estas especies y de Phylacia turbinata, recientemente reportada para Argentina. Además se presenta un mapa con la distribución de los taxones conforme a sus registros en el Neotrópico.
\end{abstract}

Palabras clave: Camillea, Nemania, Micogeografía.

\section{INTRODUCCIÓN}

A principios y mediados del siglo $\mathrm{XX}$ Spegazzini y Dennis realizaron los primeros estudios de la familia Xylariaceae en Argentina (Sir et al., 2012a); pero las investigaciones referentes a esta familia fueron retomadas a fines de los años 90. Desde entonces el conocimiento de la diversidad de los hongos xilariáceos en el país se ha incrementado notablemente. Hasta el momento los aportes más significativos se deben principalmente a las numerosas exploraciones micológicas realizadas en las selvas de montañas

${ }^{1}$ Fundación Miguel Lillo, CONICET, Laboratorio Criptogámico, Miguel Lillo 251, San Miguel de Tucumán 4000, Tucumán, Argentina.

${ }^{2}$ Área de Protección Forestal-CIEFAP, CONICETSecretaría de Ciencia, Tecnología e Innovación Productiva de Chubut, Ruta 259 Km 16,24 CC 14, Esquel (9200), Chubut, Argentina.

${ }^{3}$ INMIBO CONICET, FCEyN, UBA, Ciudad Universitaria, Pab II, $4^{\text {to }}$ Piso, CP1428EHA, Buenos Aires, Argentina.

Autor para correspondencia: sirestebanbenjamin@gmail. com del Noroeste (NOA) por Hladki \& Romero (2001; 2003; 2005; 2007; 2009a, d; 2010) y Sir et al. (2012a, b, c; 2013; 2015; 2016). Sin embargo, los aportes referidos a las selvas del Nordeste (NEA) argentino son aún escasos (Wright \& Wright, 2005; Hladki \& Romero, 2009b, c; Hladki \& Capdet, 2010; Grosso Dalúz \& Hladki, 2013). Por tal motivo este trabajo tiene como objetivo presentar dos nuevos registros para Argentina de especies xilariáceas, una de ella coleccionada en el NEA: Camillea scriblita (Mont.) Læssøe, J.D. Rogers \& Whalley y la otra, Nemania immersidiscus Van der Gucht, Y.M. Ju \& J.D. Rogers, coleccionada en el NEA y NOA. Ambas especies son descriptas y fotografiadas, presentándose además un mapa con la distribución de cada una, inferida a partir de los registros para el Neotrópico. Adicionalmente se presenta la descripción, distribución y fotografías de Phylacia turbinata (Berk.) Dennis, recientemente incluida en el listado de especies identificadas para la zona de Oberá, provincia de Misiones (Grassi et al., 2016). 


\section{Materiales y Métodos}

Los ejemplares fueron obtenidos durante exploraciones fúngicas llevadas a cabo en un relicto de Selva Paranaense (Centro de Investigaciones Antonia Ramos-CIAR, Municipio Campo Ramón, $27^{\circ} 26^{\prime} \mathrm{S}$ y $54^{\circ} 55^{\prime} \mathrm{O}$, Oberá, Misiones) durante el mes de febrero de 2015. Además, se estudiaron especímenes coleccionados en Las Yungas del NOA (Parque Nacional Baritú-Salta, $22^{\circ} 35^{\prime} \mathrm{S}$ y $64^{\circ} 37^{\prime} \mathrm{O}$ y Parque Sierras San Javier-Tucumán, 26 $6^{\circ} 43^{\prime} \mathrm{S}$ y $\left.65^{\circ} 22^{\prime} \mathrm{O}\right)$ y materiales depositados en los herbarios LIL y LPS (Thiers, 2016).

El estudio macroscópico y microscópico de los teleomorfos se realizó siguiendo técnicas convencionales(Hladki, 2007). Para las preparaciones microscópicas se utilizaron agua destilada estéril y solución de $\mathrm{KOH} 3-5 \%$, como medios de montaje y reactivo de Melzer para testear la reacción amiloide del aparato apical.

Para el estudio de las características en cultivo se utilizaron las técnicas de aislamiento, medios y condiciones de cultivo descriptas en Hladki (2007).

Las colecciones fueron depositadas en los Herbario BAFC y LIL, y el cultivo obtenido en el Cepario LIL.

\section{Resultados y Discusión}

\section{Tratamiento taxonómico}

Camillea scriblita (Mont.) Læssøe, J.D. Rogers \& Whalley; Mycol Res. 93: 143 (1989). Tipo: Guyana Francesa, Leprieur 1162 (K, holotipo) Fig. 1, 4.

Teleomórfo. Estromas elípticos irregulares, errumpentes, superficiales, efusos aplanados a levemente convexos, negros, carbonosos, 6-20 x 6-9 mm, 1-1,2 mm de espesor. Ascomas periteciales ovoides $0,8-1 \times 0,4-0,7 \mu \mathrm{m}$. Ostíolos umbilicados rodeados por un anillo prominente, ubicados en áreas circulares hundidas coalescentes poco profundas. Ascos 8-esporados, cilíndricos; 182-311 $\mu \mathrm{m}$ de long. total, porción esporífera 145-196 x 7,5-10 $\mu \mathrm{m}$, pie 25-115 $\mu \mathrm{m}$ de largo, aparato apical IK+, azul, ligeramente cupulado, 2,9-5,8 x 3,7-6 $\mu \mathrm{m}$. Paráfisis filiformes tabicadas, abundantes. Ascosporas amarillas claras, unicelulares, inequiláteras, fusiformes, $(15,5)$ 18,0-24,0 $(29,0) \times(5,8) 6,3-7,9$
$(9,5) \mu \mathrm{m}(\mathrm{N}=60 ; \mathrm{Me}=21,0 \times 7,0 \mu \mathrm{m})$, episporio ornamentado con pequeños poros al microscopio óptico. Anamorfo no observado.

Cultivo. Colonia en Agar Avena cubriendo la placa de Petri de $9 \mathrm{~cm}$ de diám. en 3 semanas, al principio el micelio blanco y algodonoso, tiñe al medio de color castaño claro, luego el micelio adopta un aspecto afelpado, de color blanco amarillento en el borde y castaño verdoso en el centro, azonado y de margen regular. Reverso castaño oscuro. No se observaron estructuras de reproducción asexual.

Distribución. Camillea scriblita es considerada una especie pobremente representada en el Neotrópico (Hastrup \& Laessoe, 2009), ha sido coleccionada en Brasil, Colombia, Ecuador y Guyana Francesa (Læssøe et al., 1989; Hastrup \& Læssøe, 2009). Los materiales aquí presentados constituyen el límite más austral de su distribución (Tabla 1 y Fig. 4).

Comentarios. El género Camillea Fr. se caracteriza por sus estromas bipartitos con ectostroma dehiscente y ascosporas amarillentas con episporio ornamentado y sin surco germinativo. Camillea sulcata (Starbäck) Lloyd era el único representante del género conocido para el país (Læssøe et al., 1989), sin embargo, recientemente Grassi et al. (2016) incluyeron a C. fossulata (Mont.) Læssøe, J.D. Rogers \& Whalley para la micobiota de Misiones.

Las colecciones argentinas cuentan con las características típicas de C. scriblita, pero con ascosporas que presentan un rango más amplio de tamaño $(15,5-29,0 \times 5,8-9,5 v s 22-27 \times 6-9 \mu \mathrm{m})$ que aquellas descriptas por Miller (1961).

Material estudiado. ARGENTINA. Prov. Misiones: Depto. Oberá, "Centro de Investigaciones Antonia Ramos", sobre ramas de dicotiledóneas sin identificar, 24-II-2015, Sir No. 777, 778 (LIL, BAFC); 25-II-2015, Sir No. 779, 780 (LIL).

Nemania immersidiscus Van der Gucht, Y.M. Ju \& J.D. Rogers; Mycotaxon 55: 550 (1995). Tipo: Papúa Nueva Guinea, Sepik Oriental, entre Marienberg y Bien, sobre madera muerta, 16-V1992, K. Van der Gucht \& L. De Meester 92-985 (GENT, holotipo). Fig. 2, 4. 

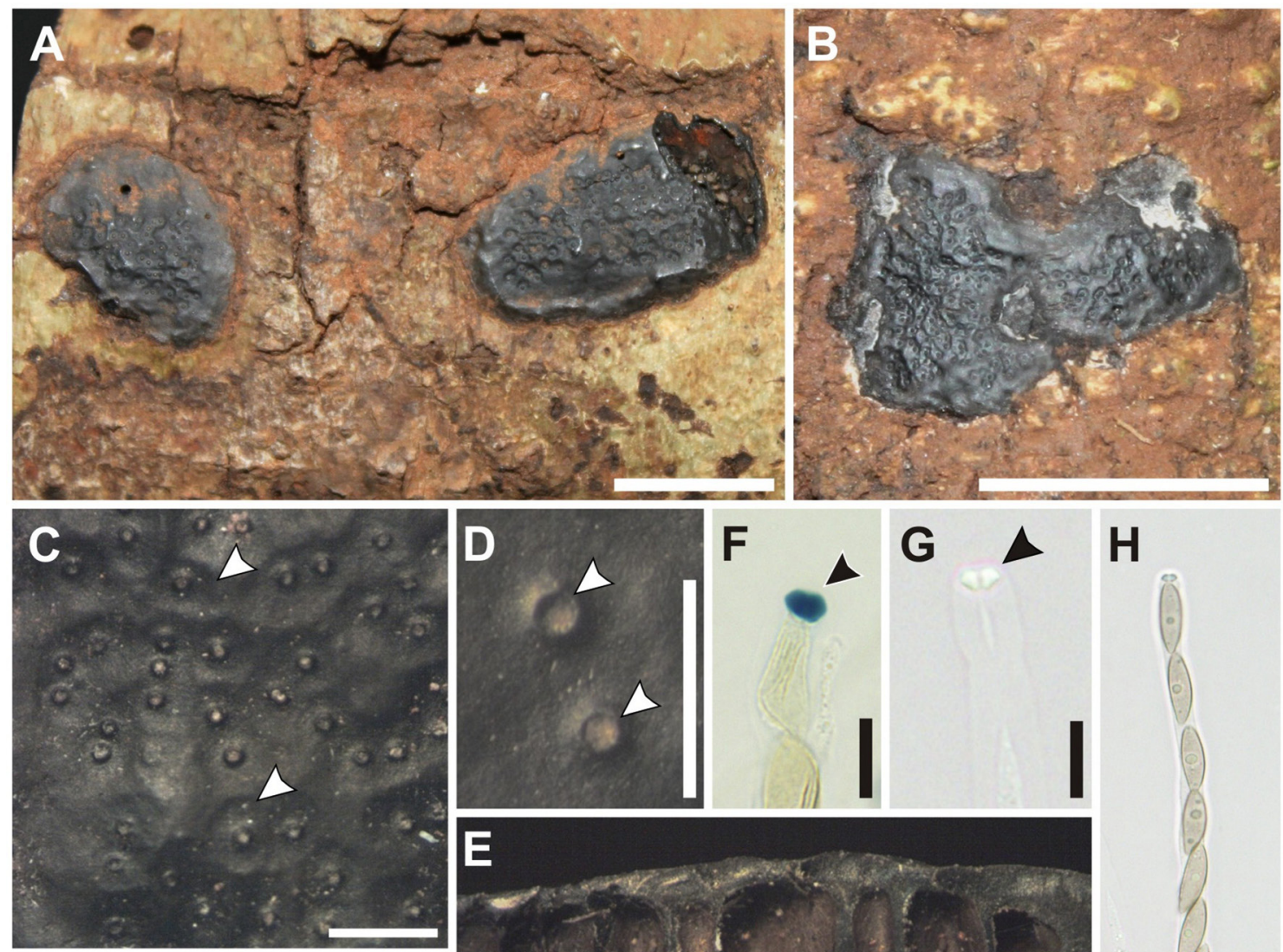
Bol. Soc. Argent. Bot. 52 (3) 2017

Tabla 1. Registros neotropicales de las especies tratadas.

\begin{tabular}{|c|c|c|c|}
\hline Especie & País & localidad & Referencia \\
\hline \multirow{5}{*}{ Camillea scriblita } & Argentina & Misiones & este estudio \\
\hline & Brasil & Rio de Janeiro & Læssøe et al. (1989) \\
\hline & Colombia & sin dato & Miller (1961) \\
\hline & Ecuador & Sucumbíos & Hastrup \& Læssøe (2009) \\
\hline & Guyana Francesa & sin dato & Læssøe et al. (1989) \\
\hline \multirow{5}{*}{ Nemania immersidiscus } & Argentina & Misiones & este estudio \\
\hline & & Salta & este estudio \\
\hline & & Tucumán & este estudio \\
\hline & Guyana & sin dato & Van der Gucht (1995) \\
\hline & Panamá & Chiriquí & Carmona et al. (2009) \\
\hline \multirow{10}{*}{ Phylacia turbinata } & Argentina & Misiones & Grassi et al. (2016) \\
\hline & Brasil & Paraná & Cruz (2015) \\
\hline & & Bahía & Dennis (1957) \\
\hline & & Rio Grande del sur & Dennis (1957) \\
\hline & & Santa Catarina & Hladki \& Romero (2009a) \\
\hline & & San Pablo & Medel et al. (2006) \\
\hline & & Amazonas & Silveira \& Rodrigues (1985) \\
\hline & Méjico & Puebla & Perez-Silva (1972) \\
\hline & & Veracruz & Perez-Silva (1972) \\
\hline & Paraguay & Guarapí & Hladki \& Romero (2009a) \\
\hline
\end{tabular}

rodeada por un área circular 0,09-0,15 mm. Ascos 8-esporados, cilíndricos, 165-282 $\mu \mathrm{m}$ de long. total, porción esporífera $115-131$ x 6,5-8,4 $\mu \mathrm{m}$, pie 91$152 \mu \mathrm{m}$, aparato apical IK+, en forma de sombrero invertido 2-2,6 x 1,8-2,1 $\mu \mathrm{m}$. Ascosporas castañas oscuras, unicelulares, elipsoidales inequiláteras, con extremos redondeados angostos, $(11,6)$ 12,4$13,9(14,9) \times(4,7) 5-6(6,4) \mu \mathrm{m}(\mathrm{N}=60, \mathrm{Me}=13,1$ x $5,4 \mu \mathrm{m})$, con surco germinativo recto tan largo como la espora en la cara más plana. Anamorfo no observado.

Distribución. Carmona et al. (2009) consideran a $N$. inmersidiscus de distribución pantropical, aunque solo se conocían especímenes coleccionados en ambientes netamente tropicales: Guyana, Hawái, Panamá y Papúa Nueva Guinea. Este nuevo registro amplía el área de distribución de la especie hacia el cono sur (Tabla 1, Fig. 4).
Comentarios. En Argentina, el género Nemania Gray estaba representado por Nemania caries (Schwein.) Y.M. Ju \& J.D. Rogers, $N$. confluens (Tode) Læssøe \& Spooner, $N$. confluens var. microspora Catania \& A. I. Romero, $N$. creoleuca (Speg.) Y.M. Ju \& J.D. Rogers, $N$. effusa (Nitschke) Pouzar, N. latissima (Speg.) Y.M. Ju \& J.D. Rogers, N. serpens (Pers.) Gray, y N. diffusa (Sowerby) Gray (Hladki \& Romero, 2006; 2009c; Catania, 2009; Catania \& Romero, 2010). N. immersidiscus es similar a $N$. circostoma (Speg.) Y.M. Ju \& J.D. Rogers (LPS 1966), de la cual se diferencia por el tamaño del disco ostiolar $(0,1-0,15$ vs $0,2-0,3 \mu \mathrm{m}$ diám.) $\mathrm{y}$ de las ascosporas (12,5-15 x 5-6 vs 15-19 x 7-8 $\mu \mathrm{m}$ ). Nemania bipapillata (Berk. \& M.A. Curtis) Pouzar es otra especie que comparte ciertos caracteres con $N$. immersidiscus, pero este taxón no presenta el disco ostiolar hundido y sus 


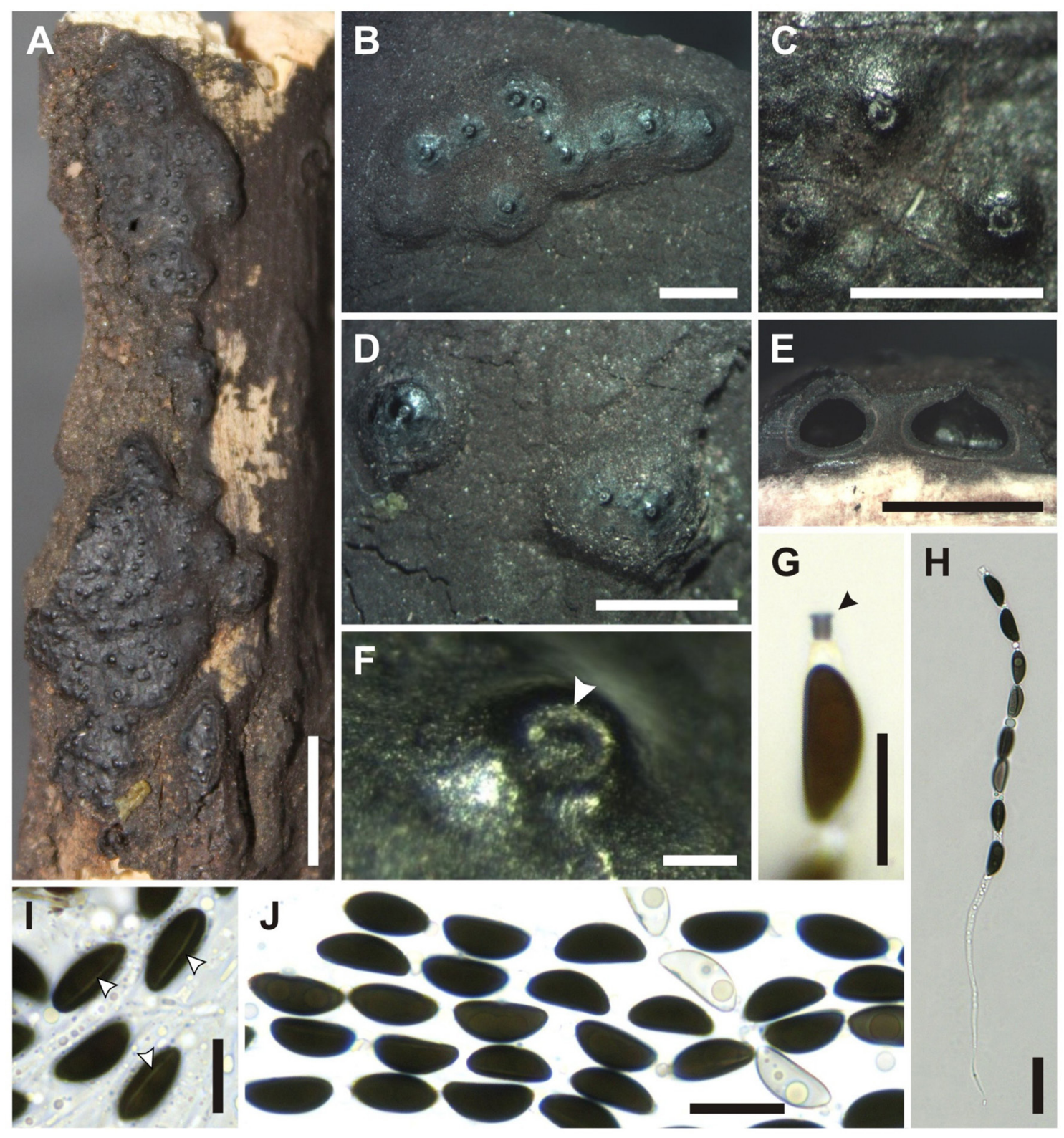

Fig. 2. Nemania immersidiscus. A: hábito del estroma. B, C y D: detalle de estromas. E: ascoma peritecial en sección vertical. F: detalle del disco ostiolar hundido (flecha). G: aparato apical amiloide (flecha). H: asco. I: ascosporas mostrando surco germinativo recto (flechas). J: ascosporas. Las escalas son indicadas por las barras ( $A=10 \mathrm{~mm}$; $B-E=2 \mathrm{~mm} ; \mathrm{F}=0,1 \mathrm{~mm} ; \mathrm{G}, \mathrm{H}=20 \mu \mathrm{m} ; \mathrm{I}, \mathrm{J}=10 \mu \mathrm{m}$ ).

ascosporas son más cortas (10,5-13,5 x 4,5-6 vs 12,5-15 x 5-6 $\mu \mathrm{m}$ ) (Van der Gucht, 1995).

Se intentaron aislamientos sin obtener resultados satisfactorios.
Material estudiado. ARGENTINA. Prov. Misiones: Dpto. Oberá, "Centro de Investigaciones Antonia Ramos", sobre madera descompuesta, 25II-2015, Sir No. 781 (LIL). Prov. Salta: Depto. Santa Victoria, Parque Nacional Baritú, Camino 
Bol. Soc. Argent. Bot. 52 (3) 2017



Fig. 3. Phylacia turbinata. A y B: vistas de estromas en sustrato. C: detalle del estroma en vista lateral. D: detalle del estroma en vista superior. E: estroma en vista superior mostrando la dehiscencia del mismo (flecha). F: sección vertical del estroma mostrando la posición de los ascomas periteciales (flecha). G: ascosporas. Las escalas son indicadas por las barras ( $A=10 \mathrm{~mm} ; \mathrm{B}-\mathrm{F}=5 \mathrm{~mm} ; \mathrm{G}=10 \mu \mathrm{m})$.

de termas, sobre rama con corteza, 27-VI-2013, Sir No. 491 (LIL). Prov. Tucumán: Depto. Yerba Buena, Parque Sierras de San Javier, La Cascada, sobre rama con corteza, 7-V-2013, Sir \& Hladki
No. 792 (LIL). Nemania circostoma: CHILE. Prov. Valparaíso: Depto. Quilpúe, Los Perales, sobre madera, 1918, Spegazzini (LPS 1966, Holotipo de Hypoxylon circostomum Speg.). 


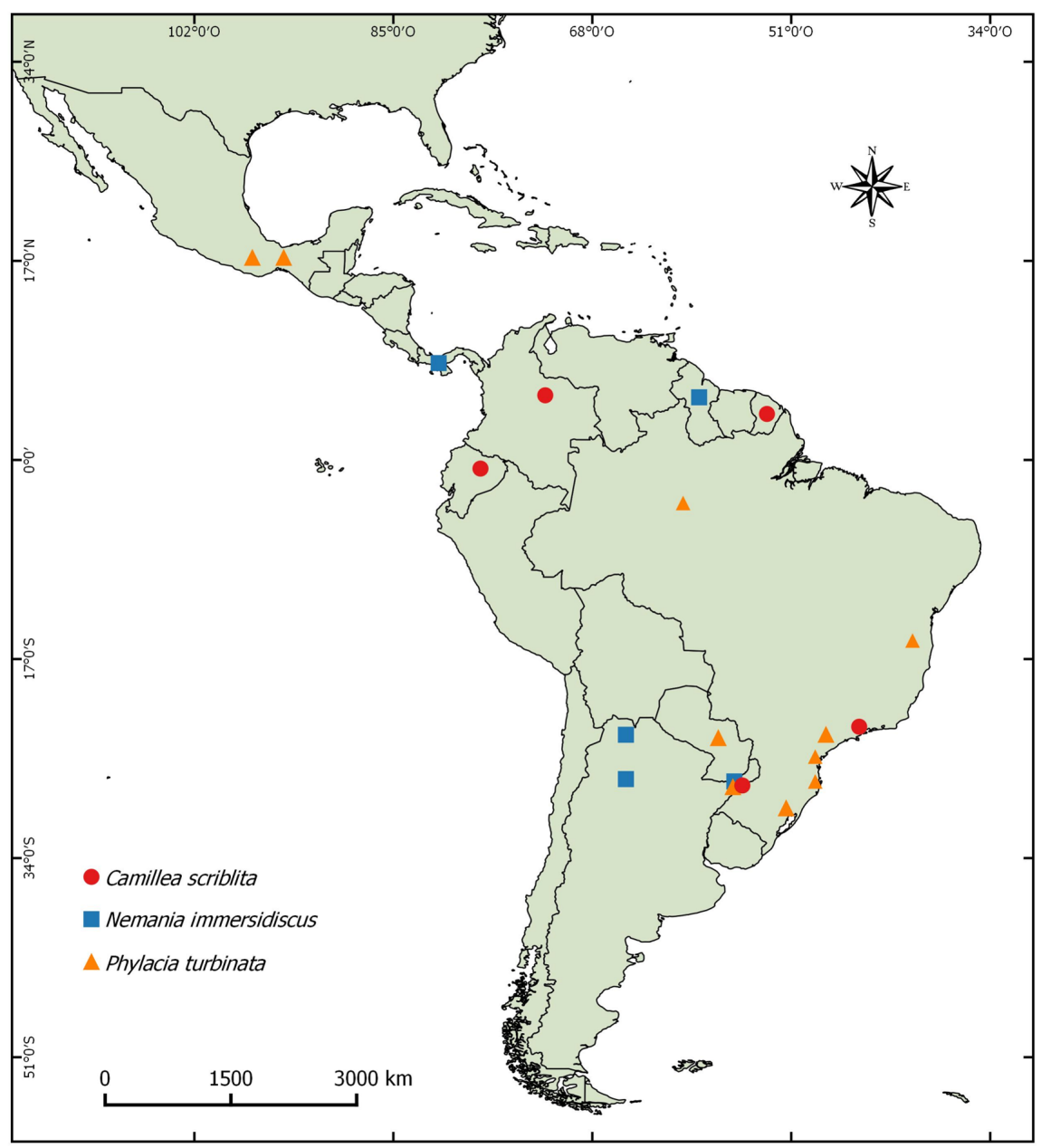

Fig. 4. Mapa de distribución de Camillea scriblita, Nemania immersidiscus y Phylacia turbinata en el Neotrópico, basado en los registros de cada especie que figuran en la bibliografía consultada.

Phylacia turbinata (Berk.) Dennis; Kew Bull. 12 (2): 323 (1957). Tipo: Brasil, Bahía, HMS Challenger Expedition, 1973 (K, holotipo). Fig. 3, 4.

Teleomórfo. Estromas solitarios a gregarios, estipitados o sésiles, 5-7,5 $\mathrm{mm}$ de alto x 3-12 $\mathrm{mm}$ diám.; turbinados, superficie castaño cobriza a negra, lisa a levemente rugosa, formada por tejido carbonoso, porción apical desintegrándose en forma circular a irregular a la madurez exponiendo la masa de esporas. Ascomas periteciales no observados. Ascos no observados. Ascosporas castañas claras elipsoidales, oblongas, con extremos redondeados anchos (9) 11,2-14,5 (15) x (5,4) 6,0-7 (7,7) $\mu \mathrm{m}(\mathrm{N}=$ $30, \mathrm{Me}=13,1 \times 6,6 \mu \mathrm{m})$, episporio liso. Anamorfo no observado.

Distribución. Phylacia turbinata es una especie ampliamente distribuida en el Neotrópico, desde Méjico hasta el sur de Brasil (Medel et al., 2006). Particularmente, la mayoría de los reportes sudamericanos corresponden a materiales coleccionados en la Mata Atlántica (Tabla 1).

Comentarios. Phylacia Lév. se caracteriza por sus estromas negros y carbonosos con ascomas 
cleistoteciales, ascos globosos o claviformes evanescentes y ascosporas elipsoidales castañas pálidas. En Argentina solo se conocía la presencia de . globosa Lév. para el NEA (Dennis, 1957) y el NOA (Hladki, 2004).

Las colecciones argentinas de $P$. turbinata fueron encontradas creciendo sobre Citrus sp. y nuestras observaciones revelaron ascosporas de mayor tamaño (9-15 x 5,4-7,7 vs 11-13 x 5-6 $\mu \mathrm{m}$ ) en comparación con la descripción aportada por Dennis (1957). Sin embargo, al examinar el material de Brasil (Hladki No. 2392), encontramos que ambos comparten un similar rango en el tamaño de ascosporas.

Se intentaron aislamientos sin obtener resultados satisfactorios.

Material estudiado. ARGENTINA. Prov. Misiones: Depto. Oberá, Centro de Investigaciones Antonia Ramos, sobre Citrus L. en pie, 25-II2015, Sir No. 785-787 (LIL). BRASIL. Prov. Santa Catalina: Depto. Florianópolis, sobre restos de madera, I-2001, Hladki No. 2392 (LIL). PARAGUAY, Guarapí, sobre Citrus aurantium L., 1879, Balansa No. 3417 (LPS 1944, Holotipo de $H$. turbinatum var. guaraniticum Speg.).

\section{Agradecimientos}

Los autores desean agradecer a la Fundación Bosques Nativos Argentinos para la Biodiversidad (FBNA) y al Centro de Investigaciones Antonia Ramos (CIAR) por permitirnos realizar las campañas de recolección en su predio. También agradecemos a la Administración de Parques Nacionales, a la Fundación Miguel Lillo y al Consejo Nacional de Investigaciones Científicas y Técnicas (CONICET). Este trabajo surgió en el marco del curso de posgrado: "Micología a campo: Identificación de macrohongos degradadores de madera" realizado en febrero de 2015.

\section{Biblografía}

CARMONA, A., J. FOURNIER, C. WILLIAMS \& M. PIEPENBRING. 2009. New records of Xylariaceae from Panama. North American Fungi 4: 1-11.

CATANiA, M. D. V. 2009. Diversidad de Hymenoascomycetes y Loculoascomycetes sobre
Podocarpus parlatorei Pilg. en las provincias de Tucumán y Catamarca. Tesis de Doctorado, Universidad Nacional de Tucumán, San Miguel de Tucumán.

CATANIA, M. D. V. \& A. I. ROMERO. 2010. Micromicetes asociados a la corteza y madera de Podocarpus parlatorei (Podocarpaceae) en la Argentina: VI. Ascomycota. Darwiniana, nueva serie 48: 123-140.

CRUZ, K. S. 2015. Xylariaceae (Ascomycota) no parque estadual de São Camilo, Palotina, PR. Tesis de Maestría, Universidade Federal do Paraná, Curitiba.

DENNIS, R. W. G. 1957. Further notes on tropical American Xylariaceae. Kew Bull. 12: 297-332.

GRASSI, E. M., G. M. ROMANO \& N. F. SCHENONE. 2016. Macrohongos presentes en un área de manejo regenerativo de bosque de Mata Atlántica (Misiones, Argentina). Bol. Soc. Argent. Bot. 51: 223-233.

GROSSO DALÚZ, L. \& A. I. HLADKI. 2013. El género Hypoxylon (Xylariaceae-Ascomycota) en el nordeste argentino (NEA). Bol. Soc. Argent. Bot. 48, supl.: 212.

HASTRUP, A. C. S. \& T. LÆSSØE. 2009. Camillea (Xylariaceae, Ascomycota), including two new species, along a trans-Andean altitude gradient in Ecuador. Mycol. Prog. 8: 305-316.

HLADKI, A. I. 2004. Contribución al estudio de las Xylariaceae de la República Argentina, los géneros Daldinia, Entonaema y Phylacia para Tucumán. Lilloa 41: 9-22.

HLADKI, A. I. 2007. La familia Xylariaceae (orden Xylariales), en la provincia de Tucumán. Tesis de Doctorado, Universidad Nacional de Tucumán, San Miguel de Tucumán.

HLADKI, A. I. \& M. CAPDET. 2010. Biodiversidad de Xylariaceae (Ascomycota) presentes en el Nordeste Argentino (NEA). $X$ Congreso Latinoamericano de Botánica, La Serena, Chile, pág. 888.

HLADKI, A. I. \& A. I. ROMERO. 2001. The genus Kretzschmaria from Tucumán (Argentina). Mycotaxon 79: 481-496.

HLADKI, A. I. \& A. I. ROMERO. 2003. Two new species of Stilbohypoxylon and the taxonomic positions of Hypoxylon cyclopicum, H. chionostomum, and Anthostoma chionostoma. Sydowia 55: 65-76.

HLADKI, A. I. \& A. I. ROMERO. 2005. Contribución al estudio de las Xylariaceae de la República Argentina. III) Nuevos registros del género Xylaria. Lilloa 42: 47-68.

HLADKI, A. I. \& A. I. ROMERO. 2006. Revisión de las especies de Hypoxylon propuestas por Spegazzini. Lilloa 43: 45-60.

HLADKI, A. I. \& A. I. ROMERO. 2007. Primeras citas del género Xylaria (Ascomycota-Xylariaceae) para la República Argentina. Darwiniana 45: 28-44. 


\section{E. B. Sir et al. - Xylariales en Argentina}

HLADKI, A. I. \& A. I. ROMERO. 2009a. Taxonomic and nomenclatural aspects of Hypoxylon taxa from Southern South America proposed by Spegazzini. Mycologia 101: 733-744.

HLADKI, A. I. \& A. I. ROMERO. 2009b. El género Kretzschmaria (Xylariaceae - Ascomycota) en el Noreste Argentino (NEA). Bol. Soc. Argent. Bot. 44, supl.: 120.

HLADKI, A. I. \& A. I. ROMERO. 2009c. Xylaria berkeleyi (Xylariaceae-Ascomycota) primera cita para la República Argentina. Lilloa 46: 68-71.

HLADKI, A. I. \& A. I. ROMERO. 2009d. Novedades para los géneros Annulohypoxylon e Hypoxylon (Ascomycota-Xylariaceae) en la República Argentina. Darwiniana 47: 278-288.

HLADKI, A. I. \& A. I. ROMERO. 2010. A preliminary account of Xylaria in the Tucuman province, Argentina, with a key to species from the Northern Provinces. Fungal Divers. 42: 79-96.

LÆSSØE, T., J. D. ROGERS, \& A. J. S. WHALLEY. 1989. Camillea, Jongiella and light-spored species of Hypoxylon. Mycol. Res. 93:121-155.

MILLER, J. H. 1961. A monograph of the world species of Hypoxylon. Universidad de Georgia Press, Atenas.

MEDEL, R., J. D. ROGERS \& G. GUZMÁN. 2006. Phylacia mexicana sp. nov. and considerations of other species with emphasis on Mexico. Mycotaxon 97: 279-290.

PEREZ-SILVA, E. 1972. El género Phylacia (Pyrenomycetes) en México. Bol. Soc. Mex. Micol. 6: 9-16

SILVEIRA, V. D. \& K. F. RODRIGUES. 1985. Levantamento preliminar de Xylariaceae da Amazônia. Acta Amazonica Supl. 15: 7-27.

SIR, E. B., A. I. HLADKI, M. F. PARRADO \& A. I. ROMERO. 2012a. Biodiversity of Xylariaceae (Ascomycota) and their hosts in protected areas from Tucumán (Argentina). Kurtziana 37: 35-48.
SIR, E. B., E. KUHNERT, C. LAMBERT, A. I. HLADKI, A. I. ROMERO \& M. STADLER. 2016. New species and reports of Hypoxylon from Argentina recognized by a polyphasic approach. Mycol. Prog. 15: 1-19. DOI: 10.1007/s11557-016-1182-z.

SIR, E. B., T. C. PERERA, A. I. ROMERO \& A. I. HLADKI. 2012b. Provisional dichotomic keys for the genera and species of Xylariaceae (Ascomycota) from Tucumán, Argentina. Lilloa 49: 126-134.

SIR, E. B., T. C. PERERA, A. I. ROMERO \& A. I. HLADKI. 2012c. Novedades para el género Rosellinia (Ascomycota-Xylariaceae) en el Noroeste de la República Argentina. Bol. Soc. Argent. Bot. 47: 311-321.

SIR, E. B., T. C. PERERA, A. I. ROMERO \& A. I. Hladki. 2013. Stilbohypoxylon quisquiliarum (Ascomycota, Xylariaceae), nueva cita para la Argentina. Darwiniana, nueva serie 1: 289-294.

SIR, E. B., A. I. ROMERO \& A. I. HLADKI. 2015. A new species and a new record of Anthostomella (Xylariaceae-Ascomycota) on leaf-litter of Alnus acuminata (Betulaceae) from Argentina. Mycotaxon 130: 721-729.

THIERS, B. 2016. Index Herbariorum: A global directory of public herbaria and associated staff. New York Botanical Garden's Virtual Herbarium. Disponible en: http://sweetgum.nybg.org/science/ih/. [Acceso: 15 enero 2017].

VAN DER GUCHT, K. 1995. Illustrations and description of xylariaceous fungi collected in Papua New Guinea. Bull. Jard. Bot. Belg. 64: 219-403.

WRIGHT, J. E. \& A. M. WRIGHT. 2005. Checklist of the mycobiota of Iguazú National Park (Misiones, Argentina). Bol. Soc. Argent. Bot. 40: 23-44.

Recibido el 14 de octubre de 2016, aceptado el 24 de enero de 2017. 
